\title{
Reduction of liver stiffness following resolution of acute flares of chronic hepatitis B
}

\author{
James Fung • Ching-Lung Lai • David But • \\ Axel Hsu - Wai-Kay Seto $\cdot$ Charles Cheng • \\ Danny Ka-Ho Wong $\cdot$ Man-Fung Yuen
}

Received: 26 August 2009/Accepted: 9 July 2010/Published online: 24 July 2010

(C) The Author(s) 2010. This article is published with open access at Springerlink.com

\begin{abstract}
Background Measuring liver stiffness is becoming more popular as a non-invasive tool for assessing liver fibrosis. Aim To assess the effect of severe hepatitis B flare on liver stiffness and determine factors that correlate with liver stiffness measurements.

Methods Twenty-nine patients with severe hepatitis B flare (ALT $>10 \times$ upper limit of normal) were followed up for 1 year. Serial transient elastography was performed at the time of flare, 3-6, and 12 months after flare.

Results At the time of flare, the median liver stiffness was $16.8 \mathrm{kPa}$, with no patients having normal liver stiffness $(<6 \mathrm{kPa})$. There was a significant decrease in liver stiffness from baseline to 3-6 months (16.8 vs. $7.9 \mathrm{kPa}$, respectively, $P<0.001$ ), and a further smaller decline from 3-6 to 12 months (7.9 vs. $6.9 \mathrm{kPa}$, respectively, $P=0.039$ ). By 12 months, 10 (34\%) had normalized their liver stiffness. Baseline parameters which correlated with liver stiffness include bilirubin, ALT, albumin, prothrombin time and platelet levels (all $P<0.05$ ).

Conclusion Liver stiffness was increased in patients with severe hepatitis B flares, with return to near normal levels by 6 months. Transient elastography for proper assessment of liver fibrosis should be performed at least 6 months after flare.
\end{abstract}

Keywords Hepatitis B - Liver stiffness . Transient elastography $\cdot$ Fibroscan · Flare $\cdot$ Fibrosis

J. Fung $(\bowtie) \cdot$ C.-L. Lai · D. But · A. Hsu · W.-K. Seto ·

C. Cheng - D. K.-H. Wong · M.-F. Yuen

Department of Medicine, Queen Mary Hospital,

The University of Hong Kong, Hong Kong SAR, China

e-mail: jfung@gastro.hk

\section{Background}

Recently, there has been increasing interest in the development of non-invasive assessment of liver fibrosis. The current "gold" standard for assessment of liver fibrosis remains the liver biopsy. However, liver biopsy is an invasive procedure associated with both patient morbidity and, less commonly, mortality. In Asia, where chronic hepatitis B is endemic, there is a constant need for physicians to evaluate the severity of underlying fibrosis, so that treatment to retard disease progression can be started for eligible patients. Likewise, it is important to identify patients with underlying cirrhosis to screen for varices and hepatocellular carcinoma. Because of the high rate of nonacceptance of liver biopsy in Asian patients, they are rarely performed. Therefore, there is a need for a more acceptable method of assessment of liver fibrosis.

Liver stiffness measurement using transient elastography has become available as a non-invasive assessment of liver fibrosis. Transient elastography determines liver elasticity by measuring the velocity of a low-frequency shear wave passing through the liver. Previous studies have shown good correlation between liver stiffness and liver fibrosis, with a AUROC of greater than 0.90 for METAVIR stage 3 fibrosis or greater in patients with hepatitis C, hepatitis C co-infected with human immunodeficiency virus, chronic biliary disease, and other liver diseases [1-5]. The diagnostic accuracy of transient elastography for liver fibrosis is supported by a recently published meta-analysis [6]. Although an earlier study showed no correlation between disease activity and liver stiffness, more recent studies have shown that liver stiffness appears to be affected by underlying inflammation, as in patients with acute hepatitis flares $[7,8]$. In the present study, we aim to determine the effect of severe 
flare of hepatitis B on liver stiffness measurements over a 12-month period.

\section{Patients and methods}

This was a prospective study. All chronic hepatitis B patients, defined by hepatitis B surface antigen (HBsAg) positive for more than 6 months, with hepatitis $B$ flare of ALT greater than 10 times upper limit of normal (ULN), and admitted to Queen Mary Hospital, Hong Kong, between the period May 2006 and August 2007, were included. All patients had HBV DNA levels $>10^{5}$ copies/ $\mathrm{mL}$. Acute hepatitis A and E were excluded by serological testing. Drug-induced hepatitis was excluded by careful history taking. None of the patients included had underlying significant alcohol intake as defined by a daily intake of $>20 \mathrm{~g} /$ day. All patients gave informed consent prior to the testing for liver stiffness measurements. Patient demographics and laboratory parameters were recorded at the time of liver stiffness measurement, including age, sex, liver biochemistry, $\mathrm{HBsAg}$, hepatitis B e-antigen (HBeAg), antibody to HBeAg (anti-HBe), and serum HBV DNA levels. HBV DNA was measured using the COBAS Taqman ${ }^{\circledR}$ HBV DNA Assay (Roche Diagnostics, Branchburg, $\mathrm{NJ}$ ), with a lower detection limit of 60 copies $/ \mathrm{mL}$.

\section{Liver stiffness measurement}

Liver stiffness measurement was performed using the Fibroscan $^{\circledR}$ (EchoSens, Paris, France). This procedure has been well described previously [2]. Only patients with at least ten valid measurements and a success rate of over $60 \%$ were included. Liver stiffness scores were expressed in units of kilopascals $(\mathrm{kPa})$. This was performed in all patients at the time of admission, 3-6, and 12 months after the time of flare. A subgroup also had liver stiffness determined at 4 weeks after flare. We adopted the value of $<6.0 \mathrm{kPa}$ as normal liver stiffness, as defined by a previous study [9].

\section{Liver histology}

Five patients underwent liver biopsy during their hepatitis B flare. Three patients were admitted for hepatitis B flare outside the recruitment period (with the same inclusion and exclusion criteria as listed). The necroinflammatory scores and degree of fibrosis were assessed according to the modified hepatic activity index grading and staging system $[10,11]$.

Statistical analysis

All statistical analyses were performed using the SPSS version 14.0 (SPSS Inc, Chicago, IL). Chi-square test was used for categorical variables, and Fisher's exact test when appropriate. Continuous variables with skewed distribution were analyzed using Mann-Whitney test. Paired related data were analyzed using the Wilcoxon paired test. Correlation between liver stiffness and liver biochemistry was performed using Spearman's bivariate correlation. A $P$ value of $<0.05$ was considered statistically significant.

\section{Results}

A total of 38 patients were recruited during the study period. Five patients were excluded due to inadequate follow-up, and further four patients were excluded due to a success rate of $<60 \%$ in any one of their scans at the three different time points. Twenty-nine patients were included in the final analysis. One patient had documented evidence of fatty liver disease on ultrasonography. The patient's demographic, baseline laboratory data, and liver stiffness measurements are summarized in Table 1.

Eleven $(38 \%)$ patients were asymptomatic with abnormal liver enzymes discovered on regular blood surveillance.

Table 1 Patient demographics and baseline laboratory values

\begin{tabular}{|c|c|}
\hline Parameters & Value \\
\hline Total patients $(n)$ & 38 \\
\hline Inadequate follow-up & 5 \\
\hline Invalid scans (success $<60 \%$ ) & 4 \\
\hline Final analysis & 29 \\
\hline Sex (male) & $23(79 \%)$ \\
\hline Age (years) & $44(20-69)$ \\
\hline $\mathrm{HBeAg}$-positive & $16(55 \%)$ \\
\hline Bilirubin $(\mu \mathrm{mol} / \mathrm{L})$ & $34(10-469)$ \\
\hline Alkaline phosphatase (U/L) & $114(56-220)$ \\
\hline GGT (U/L) & $159(48-540)$ \\
\hline AST (U/L) & $524(208-1,678)$ \\
\hline ALT (U/L) & $1,464(594-2,476)$ \\
\hline Albumin (g/L) & $39(27-46)$ \\
\hline Prothrombin time (s) & $12.8(11.1-24.6)$ \\
\hline Platelets $\left(10^{9} \mathrm{~L}^{-1}\right)$ & $185(78-313)$ \\
\hline HBV DNA (copies/mL) & $\begin{array}{l}100,000,000 \\
(646,020-640,000,000)\end{array}$ \\
\hline $\begin{array}{l}\text { Liver stiffness } \\
\text { measurement }(\mathrm{kPa})\end{array}$ & $16.8(6.9-47.2)$ \\
\hline Interquartile ratio $^{\mathrm{a}}$ & $17 \%(4-47 \%)$ \\
\hline$<10 \mathrm{kPa}$ & $7(24 \%)$ \\
\hline $10-20 \mathrm{kPa}$ & $10(35 \%)$ \\
\hline$>20 \mathrm{kPa}$ & $12(41 \%)$ \\
\hline
\end{tabular}

Continuous variables expressed as median values

${ }^{\text {a }}$ Interquartile ratio $=$ interquartile range/liver stiffness. Eighty-six percent of patients had ratio $\leq 30 \%$ 
Table 2 Comparison of liver stiffness and liver biochemistry at baseline, 3-6, and at 12 months

\begin{tabular}{lcll}
\hline Parameters & Baseline & $3-6$ months & 12 months \\
\hline Total bilirubin & $34(10-469)$ & $12(4-39)$ & $12(2-46)$ \\
ALT (U/L) & $1,464(594-2,476)$ & $30(11-66)$ & $21(13-46)$ \\
Albumin (g/L) & $39(27-46)$ & $44(36-52)$ & $45(38-49)$ \\
$\begin{array}{l}\text { Liver stiffness } \\
\quad 16 \text { ( })\end{array}$ & $16.8(6.9-47.2)^{\dagger}$ & $7.9(4.6-20.4)^{\dagger *}$ & $6.9(3.3-23.6)^{*}$ \\
\hline
\end{tabular}

Continuous variables expressed as median values

$\dagger P<0.001$ comparing liver stiffness value between baseline and 3-6 months

* $P=0.039$ comparing liver stiffness value between 3-6 and 12 months

Eight (28\%) patients presented with jaundice, and ten (35\%) patients presented with non-specific viral symptoms without jaundice. None of the patients had normal liver stiffness $(<6.0 \mathrm{kPa})$ at the time of hepatitis flare; the median liver stiffness was $16.8 \mathrm{kPa}$ (range 6.9-47.2). Twenty-four (83\%) patients were treated with oral nucleoside/nucleotide analogues.

\section{At 3-6 months follow-up}

At 3-6 months, there was a significant decline in liver stiffness from a median baseline level of $16.8 \mathrm{kPa}$ (range $6.9-47.2)$ to $7.9 \mathrm{kPa}$ (range 4.6-20.4); $(P<0.001)$. Eight $(28 \%)$ patients had normalized their liver stiffness results, as defined by a liver stiffness measurement of $<6 \mathrm{kPa}$, whereas no patients at baseline had normal liver stiffness measurement. Patients who normalized their liver stiffness at 3-6 months had significantly lower liver stiffness at baseline compared to patients with abnormal liver stiffness at 3-6 months (9.0 vs. $20.4 \mathrm{kPa}$, respectively, $P=0.021$ ). There was no significant difference in the age, and baseline levels of bilirubin, ALT, platelets, albumin, and prothrombin time, between patients with or without normal liver stiffness at 3-6 months. Twenty-six (90\%) had normalized their ALT, with the remaining three patients only having minimally elevated ALT of less than twice the upper limit of normal. The results are summarized in Table 2.

\section{At 12 months follow-up}

At 12 months, the median liver stiffness was $6.9 \mathrm{kPa}$ (range 3.3-23.6), which was significantly lower when compared to liver stiffness at 3-6 months after flare $(P=0.039)$. Ten patients $(34 \%)$ had normalized their liver stiffness after 1 year. All patients had normalized their ALT at 12 months after initial flare. The results are summarized in Table 2. Only one patient had HBV DNA level $>10^{5}$ copies/mL $(101,850$ copies $/ \mathrm{mL})$, and $59 \%$ had undetectable HBV DNA.

Five $(17 \%)$ patients had the diagnosis of liver cirrhosis at 12 months, defined by a liver stiffness value of $>10.3 \mathrm{kPa}$ [12]. Baseline factors associated with the diagnosis of cirrhosis at 12 months included a higher ALP level (164 vs. $107 \mathrm{U} / \mathrm{L}$, respectively, $P=0.027$ ) and liver stiffness (40.3 vs. $14.0 \mathrm{kPa}$, respectively, $P=0.016$ ), and lower albumin level (35 vs. $40 \mathrm{~g} / \mathrm{L}$, respectively, $P<0.001$ ), compared to those patients that did not have cirrhosis. The baseline age, bilirubin, AST, ALT, prothrombin time, platelets, and HBV DNA levels were not significantly different between these two groups.

The median liver stiffness measurements at baseline, 3-6, and at 12 months are summarized in Fig. 1. The serial liver stiffness measurements of every individual patient are shown in Fig. 2. The changes in liver stiffness between different time points are shown in Fig. 3.

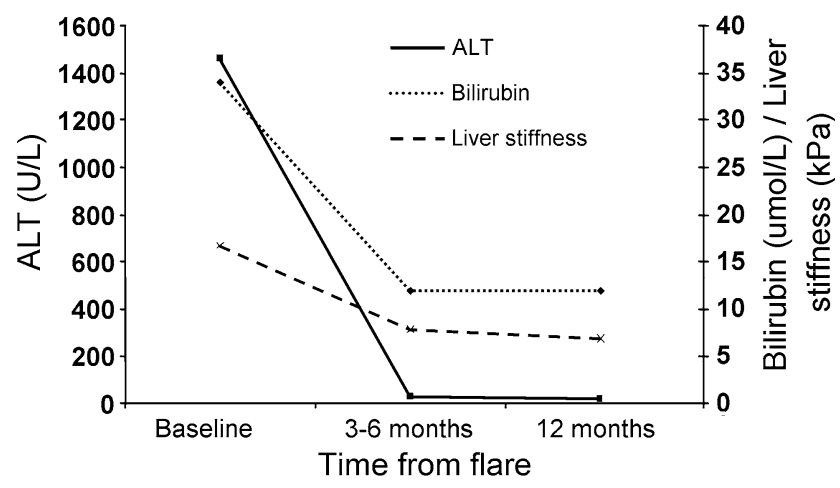

Fig. 1 Median levels of bilirubin, ALT, and liver stiffness at baseline, 3-6 and 12 months

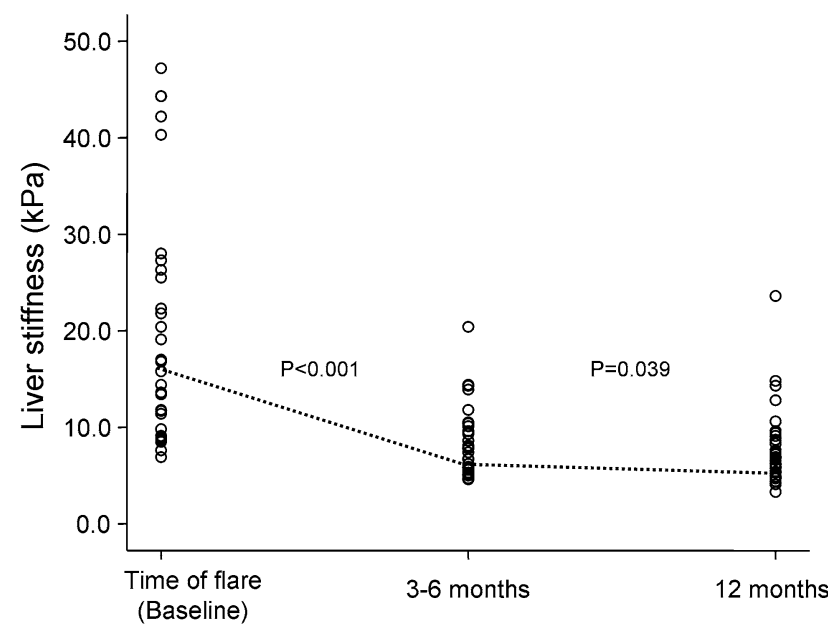

Fig. 2 Distribution of liver stiffness of all patients at baseline, 3-6, and at 12 months 


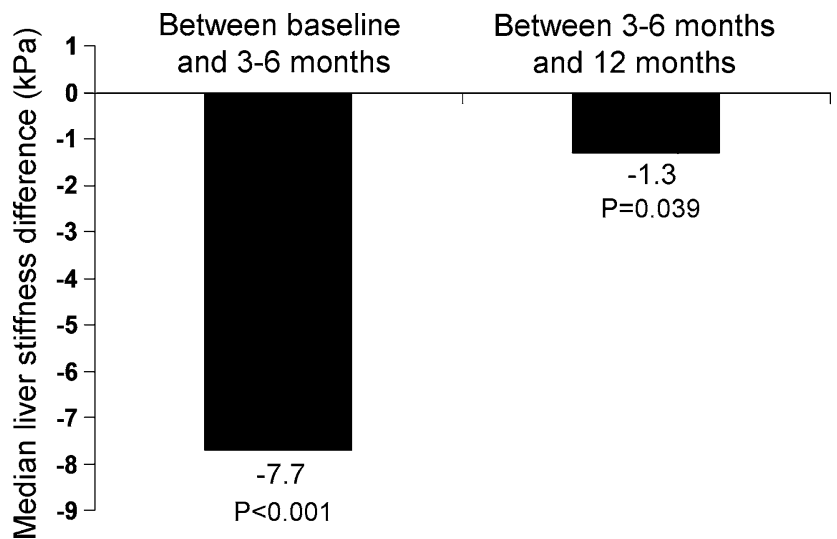

Fig. 3 Median reduction in liver stiffness between baseline and 3-6, and between 3-6 and 12 months

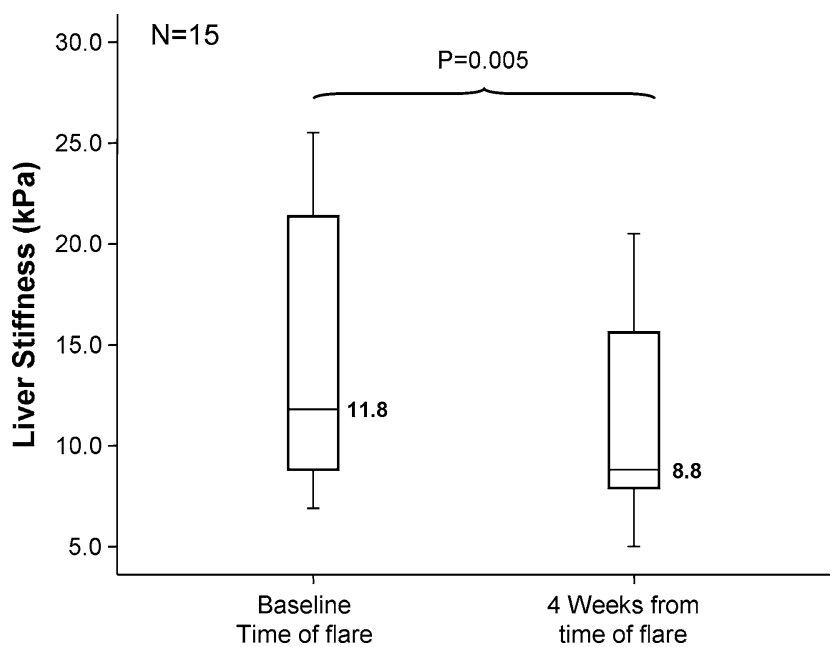

Fig. 4 Median liver stiffness at baseline and at 4 weeks (subgroup analysis of 15 patients)

Further follow-up beyond 12 months

Of the 29 patients, 25 were available for repeat liver stiffness measurement at the time of last follow-up, beyond the 12 months study period, with a median time of 34 months from the time of flares. There was a further decline in liver stiffness of $1.0 \mathrm{kPa}(P=0.02)$ between the last follow-up time and at 12 months. None of these 25 patients had liver stiffness measurements of $>10.3 \mathrm{kPa}$.

Early decline in liver stiffness

A subgroup of 15 patients (52\%) was available at week 4 for early follow-up and had liver stiffness measurements performed at this visit. There was a significant decline at 4 weeks compared to baseline ( 8.8 vs. $11.8 \mathrm{kPa}$, respectively, $P=0.005$ ). At 4 weeks, the median ALT was $103 \mathrm{U} / \mathrm{L}$ (range 14-437), compared to the baseline level of
Table 3 Correlation of baseline parameters with liver stiffness

\begin{tabular}{lrr}
\hline Parameters & Correlation coefficient $^{\mathrm{a}}$ & $P$ value \\
\hline Age & -0.221 & 0.249 \\
HBeAg status & 0.310 & 0.102 \\
Bilirubin & 0.470 & 0.010 \\
Alkaline phosphatase & 0.137 & 0.478 \\
GGT & -0.335 & 0.110 \\
AST & 0.353 & 0.060 \\
ALT & 0.371 & 0.047 \\
Albumin & -0.471 & 0.010 \\
Prothrombin time & 0.687 & $<0.001$ \\
Platelet & -0.532 & 0.004 \\
HBV DNA & 0.310 & 0.102 \\
\hline
\end{tabular}

a Spearman's correlation coefficient

1,532 U/L (range 612-2476). The results are shown in Fig. 4. Of those patients that had early decline in liver stiffness, there was no difference in the HBV DNA levels at 12 months when compared to those patients that did not have an early decline (144 vs. 60 copies $/ \mathrm{mL}, P=0.17$ ). Therefore, early decline in liver stiffness was not predictive of viral suppression after 1 year.

Correlation of baseline parameters and liver stiffness

The baseline parameters and their correlation are summarized in Table 3. The age, HBeAg status, levels of ALP, GGT, AST and HBV DNA did not significantly correlate with liver stiffness. Baseline factors which correlated with liver stiffness measurements included bilirubin, ALT, albumin, prothrombin time, and platelet levels $(P=0.010$, $P=0.047, P=0.010, P<0.001$ and $P=0.004$, respectively). The correlation co-efficient between baseline parameters and liver stiffness at 3-6 months for bilirubin, ALT, albumin, prothrombin time, and platelet levels was $0.346(P=0.066), 0.220(P=0.252),-0.458(P=0.013)$, $0.474(P=0.011)$, and $-0.227(P=0.245)$, respectively. The correlation co-efficient between baseline parameters and liver stiffness at 12 months for bilirubin, ALT, albumin, prothrombin time, and platelet levels was $0.324(P=$ $0.086), 0.090 \quad(P=0.642),-0.499 \quad(P=0.006), 0.321$ $(P=0.096)$, and $-0.110(P=0.577)$, respectively. There were also significant correlations between the liver stiffness measurement and ALT levels at 3-6 months (correlation coefficient $=0.449, P=0.014$ ) and at 12 months (correlation coefficient $=0.458, P=0.016$ ).

There was no significant difference in baseline liver stiffness between $\mathrm{HBeAg}$-positive and $\mathrm{HBeAg}$-negative patients ( 16.8 vs. $16.4 \mathrm{kPa}$, respectively, $P=0.942$ ). After 6 months, there was also no significant difference in liver stiffness between $\mathrm{HBeAg}$-positive and $\mathrm{HBeAg}$-negative 
Table 4 Liver stiffness, histological activity and stage of fibrosis during severe flare

${ }^{a}$ Necroinflammatory and fibrosis score according to the modified hepatic activity index grading and staging system

\begin{tabular}{llrrrll}
\hline Patient & Sex & Age & ALT & Liver stiffness (kPa) & $\begin{array}{l}\text { Necroinflammatory } \\
\text { score }(\max 18)^{\mathrm{a}}\end{array}$ & $\begin{array}{l}\text { Fibrosis score } \\
(\max 6)^{\mathrm{a}}\end{array}$ \\
\hline 1 & M & 28 & 629 & 14.3 & 4 & 1 \\
2 & M & 48 & 759 & 6.9 & 3 & 2 \\
3 & M & 21 & 899 & 8.8 & 6 & 1 \\
4 & M & 46 & 1,184 & 20.4 & 8 & 3 \\
5 & M & 24 & 673 & 34.3 & 5 & 1 \\
\hline
\end{tabular}

patients (7.3 vs. $9.6 \mathrm{kPa}$, respectively, $P=0.178$ ). There was also no significant correlation with baseline $\mathrm{HBV}$ DNA with liver stiffness at baseline and at 6 months after flares $(P=0.102$ and $P=0.272$, respectively).

\section{ALT flare and liver stiffness}

The levels of ALT were stratified into groups above 1,000, 1,500 , and above 2,000 U/L. There was no difference in liver stiffness between patients with ALT $>1,000 \mathrm{U} / \mathrm{L}$ and in those with ALT $<1,000 \mathrm{U} / \mathrm{L}(20.4$ vs. $16.3 \mathrm{kPa}$, respectively, $\quad P=0.40$ ), and in patients with ALT $>1,500 \mathrm{U} / \mathrm{L}$ compared with ALT $<1,500 \mathrm{U} / \mathrm{L}(23.9$ vs. $15.8 \mathrm{kPa}$, respectively, $P=0.201)$. There was a significant difference in patients with ALT $>2,000 \mathrm{U} / \mathrm{L}$ compared with those with ALT $<2,000 \mathrm{U} / \mathrm{L}$ (34.3 vs. $14.4 \mathrm{kPa}$, respectively, $P=0.022$ ).

\section{Antiviral therapy}

Twenty-four (83\%) patients received nucleoside/nucleotide analogues with lamivudine (4 patients), adefovir (2 patients), or entecavir (18 patients). After 3 months, there was no significant difference in median liver stiffness reduction in patients who received antiviral therapy and those that did not $(7.2$ vs. $12.4 \mathrm{kPa}$, respectively, $P=0.845)$.

\section{Liver histology during flare}

Five patients underwent liver biopsy during their inpatient admission at the time of hepatitis B flare. Two were from this study population, and three additional patients were admitted outside the recruitment time. The results are summarized in Table 4. The liver stiffness score was much higher than the expected value for the stage of fibrosis observed on histology.

\section{Discussion}

Recently, transient elastography has become available as a new method in the non-invasive assessment of liver fibrosis. Although earlier studies had been performed on Caucasian patients with chronic hepatitis $\mathrm{C}$, more recent studies have shown transient elastography to be similar in accuracy for Asian patients and in patients with chronic hepatitis B [13-17]. Initial studies have shown that liver stiffness measurement with transient elastography correlated well with underlying liver fibrosis, with good diagnostic performance [1-4]. Most of these studies have been performed on patients with chronic hepatitis $\mathrm{C}$, with less available data on chronic hepatitis B. Even less data are available with regard to liver stiffness measurement during the time of hepatitis flare.

A recent study performed by Arena et al. [8] of 18 patients with acute viral hepatitis [hepatitis $\mathrm{A}(n=7), \mathrm{B}$ $(n=8)$, and $\mathrm{C}(n=3)]$ has shown a significant correlation between liver stiffness and serum aminotransferase levels, concluding that liver stiffness measurement is not reliable in the context of acute hepatitis. In that particular study, the timing of liver stiffness measurements was performed with regard to the defined ALT levels after an episode of flare, namely at the time when ALT halved, and finally at the time when ALT was reduced to $<2 \times$ ULN. In another study performed by Sagir et al. [18] of 20 patients with hepatitis flares [hepatitis A $(n=1)$, hepatitis B $(n=8)$, toxic $(n=8)$, and autoimmune hepatitis $(n=3)]$, liver stiffness was found to be elevated in the absence of severe fibrosis or cirrhosis during the time of flare. The correlation between ALT levels and the liver stiffness measurement was done by pooling the measurements from 20 patients. The longitudinal data on liver stiffness were available in a limited number of patients. In contrast to these two previous studies, the current study looks specifically at those patients with acute severe flare of hepatitis B, followed up for 1 year, with liver stiffness measurement performed at specific time points, namely, at the time of the severe flares, 1, 3-6, and 12 months after these flares.

We identified several significant factors which correlated with liver stiffness during an acute flare, including higher bilirubin, ALT, and prothrombin time, and lower albumin and platelet levels. The baseline ALT and platelet levels, however, were not shown to be correlated with liver stiffness at 3-6 or at 12 months. By contrast, the baseline albumin level was shown to be inversely 
correlated to liver stiffness at baseline, after 3-6, and at 12 months. There was a trend for correlation with baseline bilirubin level and prothrombin time with liver stiffness at 12 months. This suggests that baseline parameters such as bilirubin, prothrombin time, and albumin may indicate underlying pre-existing damage to the liver already and is more predictive of long-term outcome rather than the severity of the ALT rise at the time of hepatitis flare. By stratifying the ALT levels, a significantly higher liver stiffness measurement was shown only for patients with ALT higher than 2,000 U/L. The significance of this remains to be defined.

Most of the decline in liver stiffness after a severe flare occurs within the first 6 months, with only further minimal reduction from 6 to 12 months. These results suggest that after an acute hepatitis B flare, liver stiffness returns close to its background level by 3-6 months, with further minimal decline over the next 6 months. This is in keeping with a larger population study of 1,315 CHB patients, whereby the median liver stiffness was found to be $6.7 \mathrm{kPa}$, which is comparable to the value of $6.9 \mathrm{kPa}$ at 12 months after flare in the present study population [13]. Although the decline in liver stiffness in our patients may be partly related to reversal of fibrosis with antiviral therapy, it is likely that the major cause of the decline was due to decrease in inflammatory activities. Liver stiffness measurements should be performed at least 6 months after resolution of severe flares (e.g. ALT $<2 \times \mathrm{ULN}$ ). If the results remain unexpectedly high, we would recommend repeating another scan in a further 6 months time, as we have shown that there is a further significant decline in liver stiffness between 3-6 and 12 months. Moreover, in those patients with a persistently high measurement at 12 months, a further scan is recommended, as in the current study, these patients had further decline in their liver stiffness at the time of last follow-up. This would give a more accurate assessment of the degree of background severe fibrosis or cirrhosis.

Whether this can be applied to other causes of severe acute flares of hepatitis, such as acute alcoholic hepatitis, autoimmune flares, acute presentation of Wilson's disease, drug-related hepatitis, or other viral hepatitis, remains to be determined. The two previous studies, having shown that liver stiffness is elevated during acute hepatitis flares from other causes, including hepatitis $\mathrm{A}$, hepatitis $\mathrm{C}$, toxic hepatitis, and autoimmune hepatitis, will provide strong evidence for this [8, 18]. Of note, an increased liver stiffness measurement after an episode of severe flare was not predictive of the presence of underlying advanced fibrosis or cirrhosis, as all patients had lowered their liver stiffness at the time of last follow-up.

Although we have shown that liver stiffness is significantly higher during the time of hepatitis flare, the nature of this elevated liver stiffness remains to be determined. Whether the higher measurements reflect truly the increase in fibrosis or reflects the architectural changes associated with necro-inflammatory activity, such as cellular infiltration, tissue necrosis, or tissue edema, is currently unknown. Furthermore, there remains the possibility that the higher liver stiffness measurements seen during an acute hepatitis flare may be artefactual, due to higher water content at the time of severe flares, rather than a true increase in liver stiffness. There is likely a complex interplay of matrix metalloproteinases and their inhibitors during the phase of acute viral hepatitis which may influence liver stiffness at the time of inflammation [19].

The true nature of the increased liver stiffness during a hepatitis flare can only be delineated by liver biopsy. We report five biopsies performed at the time of hepatitis $B$ flare, showing a higher liver stiffness value than expected for the stage of fibrosis on histology. In addition, the fact that liver stiffness was significantly reduced by as early as 1 month in our subgroup analysis, and after 3-6 months from flares, suggests that the increase in liver stiffness is not reflective of underlying fibrosis, and more as a result of inflammation. This is supported by the lack of significant fibrosis on liver histology in the current study despite having an elevated liver stiffness value, and also in a previous study [18].

Further studies are required to determine whether liver stiffness is affected by moderate inflammatory activity (e.g. ALT 2-5 times upper limit of normal). In a recently published study, increasing liver stiffness correlated well with even smaller gradients of ALT, including $<0.5 \times \mathrm{ULN}$, 0.5-1 $\times$ ULN, and 1-2 × ULN [13]. However, because of the high area under the receiver operating characteristic curve (AUROC) already achieved with transient elastography, such small increments in ALT are unlikely to affect the accuracy of the test, and smaller increments in liver enzymes may reflect underlying fibrosis rather than inflammation.

There were several limitations of the current study. First, the sample size was small; however, this is because the overall number of admissions for severe flares of CHB is relatively small. Despite the small sample size, the study was able to show significant liver stiffness changes over the different time points. Second, there were only a limited number of liver biopsies performed, as this is seldomly carried out in patients with well-documented evidence of hepatitis B flares. In addition, over one-third of the patients admitted for severe flares had elevated prothrombin time, precluding percutaneous biopsies.

In conclusion, liver stiffness is increased in chronic hepatitis B patients with severe flares, with return to near normal levels by 6 months. We recommend measuring liver stiffness to document liver fibrosis or cirrhosis in 
patients having severe hepatitis B flares should be postponed to at least 6 months after flare.

Open Access This article is distributed under the terms of the Creative Commons Attribution Noncommercial License which permits any noncommercial use, distribution, and reproduction in any medium, provided the original author(s) and source are credited.

\section{References}

1. Castera L, Vergniol J, Foucher J, et al. Prospective comparison of transient elastography, Fibrotest, APRI, and liver biopsy for the assessment of fibrosis in chronic hepatitis C. Gastroenterology 2005;128(2):343-350

2. Foucher J, Chanteloup E, Vergniol J, et al. Diagnosis of cirrhosis by transient elastography (FibroScan): a prospective study. Gut 2006;55(3):403-408

3. Corpechot C, El Naggar A, Poujol-Robert A, et al. Assessment of biliary fibrosis by transient elastography in patients with $\mathrm{PBC}$ and PSC. Hepatology 2006;43(5):1118-1124

4. Ziol M, Handra-Luca A, Kettaneh A, et al. Noninvasive assessment of liver fibrosis by measurement of stiffness in patients with chronic hepatitis C. Hepatology 2005;41(1):48-54

5. Sandrin L, Fourquet B, Hasquenoph J, et al. Transient elastography: a new noninvasive method for assessment of hepatic fibrosis. Ultrasound Med Biol 2003;29(12):1705-1713

6. Friedrich-Rust M, Ong MF, Martens S, et al. Performance of transient elastography for the staging of liver fibrosis: a metaanalysis. Gastroenterology 2008;134(4):960-974

7. Coco B, Oliveri F, Maina AM, et al. Transient elastography: a new surrogate marker of liver fibrosis influenced by major changes of transaminases. J Viral Hepat 2007;14(5):360-369

8. Arena U, Vizzutti F, Corti G, et al. Acute viral hepatitis increases liver stiffness values measured by transient elastography. Hepatology $2008 ; 47(2): 380-384$
9. Roulot D, Czernichow S, Le Clesiau H, et al. Liver stiffness values in apparently healthy subjects: Influence of gender and metabolic syndrome. J Hepatol 2008;48(4):606-613

10. Ishak K, Baptista A, Bianchi L, et al. Histological grading and staging of chronic hepatitis. J Hepatol 1995;22(6):696-699

11. Knodell RG, Ishak KG, Black WC, et al. Formulation and application of a numerical scoring system for assessing histological activity in asymptomatic chronic active hepatitis. Hepatology 1981;1(5):431-435

12. Marcellin P, De Ledinghen V, Dhumeaux D, et al. Non-invasive assessment of liver fibrosis in chronic hepatitis B using fibroscan. Hepatology 2005;42(41):715

13. Fung J, Lai CL, But D, et al. Prevalence of fibrosis and cirrhosis in chronic hepatitis B: implications for treatment and management. Am J Gastroenterol 2008;103(6):1421-1426

14. Chang PE, Lui HF, Chau YP, et al. Prospective evaluation of transient elastography for the diagnosis of hepatic fibrosis in Asians: comparison with liver biopsy and aspartate transaminase platelet ratio index. Aliment Pharmacol Ther 2008;28(1):51-61

15. Kim DY, Kim SU, Ahn SH, et al. Usefulness of Fibroscan for detection of early compensated liver cirrhosis in chronic hepatitis B. Dig Dis Sci 2009;54(8):1758-1763

16. Kim SU, Ahn SH, Park JY, et al. Liver stiffness measurement in combination with noninvasive markers for the improved diagnosis of B-viral liver cirrhosis. J Clin Gastroenterol 2009;43(3): 267-271

17. Marcellin P, Ziol M, Bedossa P, et al. Non-invasive assessment of liver fibrosis by liver stiffness measurements in patients with chronic hepatitis B. Liver Int 2009;29(2):242-247

18. Sagir A, Erhardt A, Schmitt M, et al. Transient elastography is unreliable for detection of cirrhosis in patients with acute liver damage. Hepatology 2008;47(2):592-595

19. Koulentaki M, Valatas V, Xidakis K, et al. Matrix metalloproteinases and their inhibitors in acute viral hepatitis. J Viral Hepat 2002;9(3):189-193 